\title{
Addressing the Need for Patient-friendly Medical Communications: Adaptation of the 2019 Recommendations for the Management of MPS VI and MPS IVA
}

\author{
lain A. Bruce
}

Royal Manchester Children's Hospital

\section{Fatih S. Ezgü}

Gazi University: Gazi Universitesi

\section{Christoph Kampmann}

University Children's Hospital, Johannes Guterberg Universitat

\section{Vladimir Kenis}

H. Turner National Medical Research Center for Children's Orthopedics and Trauma Surgery

\section{William Mackenzie}

Nemours/Alfred I. Dupont Hospital for Children

\section{Bob Stevens}

MPS Society

\section{Robert Walker}

Royal Manchester Children's Hospital

Christian Johannes Hendriksz ( $\nabla$ chris@fymcamedical.co.uk)

Steve Biko Academic Unit, University of Pretoria https://orcid.org/0000-0003-2179-3639

\section{Research}

Keywords: Rare diseases, Mucopolysaccharidosis, MPS IVA, MPS VI, Management guidelines, Enzyme replacement therapy, Surgery, Anesthetics, Patient engagement, Patient education

Posted Date: August 13th, 2021

DOI: https://doi.org/10.21203/rs.3.rs-801127/v1

License: (9) (1) This work is licensed under a Creative Commons Attribution 4.0 International License. Read Full License 
Version of Record: A version of this preprint was published at Orphanet Journal of Rare Diseases on March 2nd, 2022. See the published version at https://doi.org/10.1186/s13023-022-02219-7. 


\section{Abstract}

\section{Background}

Patients are the most important stakeholders in the care of any disease and have an educational need to learn about their condition and the treatment they should receive. Considering this need for patientfocused materials, we present a directed approach for mucopolysaccharidosis (MPS) VI and MPS IVA, a pair of rare, inherited diseases that affects multiple organs and parts of the body. Independent guidelines on the treatment of these diseases were recently published, providing evidence- and expertise-driven recommendations to optimize patient management. However, while healthcare providers may have the training and knowledge to understand these guidelines, patients and their caregivers can find the technical content challenging. Hence, we aimed to develop plain language summaries (PLS) of the MPS $\mathrm{VI}$ and MPS IVA guidelines with patients as the primary audience.

Results

A review of the guidelines by an expert team identified six domains of information relevant to patients: The multidisciplinary team, regular tests and check-ups, disease-modifying and supportive treatments, general anesthetics, ear-nose-throat/respiratory care, and surgeries. This information was adapted into a series of infographics specific to either MPS VI or MPS IVA, designed to appeal to patients and clearly present information in a concise manner.

Conclusions

The use of patient-friendly materials, like the infographics we have developed, has the potential to better inform patients and engage them in their care. We issue a "call to arms" to the medical community for the development of similar PLS materials in rare diseases intended to inform and empower patients.

\section{Background}

The mucopolysaccharidoses (MPS) are a varied group of rare inherited lysosomal storage disorders, in which an affected person lacks a specific enzyme that is needed to break down glycosaminoglycan (GAG) proteins. These enzymes normally act within lysosomes, the components of the cell in which protein recycling takes place $[1,2]$. Resulting from the lack of this enzyme, there is a progressive build-up of GAGs in organs and tissues of persons with MPS. This leads to the development of a broad range of signs and symptoms affecting different parts of the body, depending on the specific GAG and the site where they build up $[3,4]$. The age when MPS becomes apparent can range from early onset (in some cases prior to birth) to late onset depending on the severity of MPS an individual has [1]. Each of the seven different types of MPS demonstrates similar but nuanced symptoms, yet each type is associated with a substantial impact on patients' qualities of life and reduced life expectancies [3,5]. 
MPS VI, also known as Maroteaux-Lamy syndrome, is caused by complete or partial loss of activity of the enzyme $\mathrm{N}$-acetyl-galactosamine-4-sulfatase (arylsulfatase B, ARSB), which is involved in the breakdown of two different GAGs: dermatan sulfate and chondroitin 4-sulfate [6, 7]. Symptoms of MPS VI include decreased rate of bodily growth, coarse facial features, skeletal deformities, frequent upper-airway infections (e.g. influenza), hepatosplenomegaly (swelling of the liver and spleen), hearing loss, and joint stiffness. Patients with MPS VI also have abnormalities in heart structure and function due to the buildup of dermatan sulfate within the cardiac valves $[6,7]$.

MPS IVA, also known as Morquio A syndrome, arises from loss of activity of the enzyme $\mathrm{N}$-acetylgalactosamine-6-sulfatase (GALNS), which breaks down the GAGs keratan sulfate (KS) and chondroitin sulfate (CS) $[8,9]$. Accumulation of KS and CS occurs mainly in bone, cartilage, and the surrounding zone between cells called the extracellular matrix, leading to the development of skeletal deformities. Other symptoms can include respiratory (breathing) problems, snoring with breath holding (apnea), hearing loss, and dental abnormalities $[8,10]$. The effects of MPS IVA on the airway can be progressive and involve more than one level within the airway, from "lips to lungs".

As MPS affects multiple parts of the body, its management requires an extensive multidisciplinary (across multiple specialties) team of healthcare professionals (HCPs) who provide surgical, supportive, and disease-specific treatments that are tailored to each individual [7, 9]. Comprehensive management guidelines for HCPs have recently been published for MPS VI and MPS IVA, with the intention of enhancing patient quality of life and improving clinical- and patient-reported outcomes [7, 9]. These guidelines clearly highlight the importance of ensuring that patients and their caregivers are well informed of available treatment options as the diseases progresses, so that they can make educated decisions to undertake any therapy and/or surgery $[7,9]$.

These specific guidelines demonstrate the importance of involving patients and their caregivers in healthcare decisions, and other literature has highlighted the value of engaging these groups in the planning and conduct of research so that it addresses the key evidence gaps in the management of rare diseases $[11,12]$. However, despite the wide-reaching benefits of greater patient and caregiver knowledge and engagement, people living with rare diseases often have limited access to user-friendly, evidencebased information $[13,14]$.

To support the goal of enabling greater patient involvement in the management of their disease, scientific information needs to be more accessible, as the complex technical language often used in the medical literature can be challenging for non-scientific audiences to understand [15]. Plain language summaries (PLS), using non-technical language to describe complex medical information and concepts, have emerged as an important communication tool to enable scientific research to be more easily understood, accessible, and reach a wider audience [16].

Given the need for greater knowledge and engagement among patients with rare diseases and their caregivers, we embarked on a project to develop infographic-based PLS on the current management guidelines for MPS VI and MPS IVA [7, 9]. This article describes the methods and results of our process to 
produce the PLS, which aim to convey the key concepts and recommendations from the guidelines in easily understandable, patient-friendly language. We present this publication as a "call to arms" for the development of similar PLS for traditional HCP-targeted publications.

\section{Methods}

The methods for generating the original guidelines are summarized in their respective publications $[7,9]$. Briefly, both guidelines were developed using an anonymous modified Delphi method to identify guidance statements focused on key domains of care. The development of the guidelines was led by an independently selected steering committee made up of three patient advocates and 26 international HCPs from various disciplines with expertise in managing MPS VI or MPS IVA.

To adapt these guidelines, our team was assembled to include authors from multiple disciplines with specialist experience in treating patients with MPS, involving several authors of the original guidelines including a representative from a large patient advocacy organization. An initial review of the original guidelines was carried out to identify key areas of patient impact that could be developed into summaries. These summaries were then developed as visual infographics to engage a primary target audience of adolescent to young adult patients. Infographics were developed from a written outline, and then through typeset graphic design in iterations. At each step, the infographics were carefully reviewed and refined with input from the author team.

\section{Results}

In total, six infographic-based PLS were developed for each guideline, based on the identification of key domains with high degree of patient impact (Figs. 1-12). The infographics in Figs. 1 and 2 relate to the introduction of the multidisciplinary team (MDT), which was highlighted as a core part of MPS care in both guidelines. "Introducing your care providers: The multidisciplinary team (MDT)" summarizes the MDT concept and identifies HCPs who should be involved in key areas of care, while acknowledging that the members of this team may differ depending on the healthcare system and key HCPs. It also makes clear the importance of patient involvement in decision making.

The infographics on "Regular tests and check-ups for MPS VI/IVA" adapt information from the guidelines related to routine monitoring and assessments, highlighting the schedule of key assessments and reinforcing the need for patients to attend regular clinic visits (Figs. 3, 4). To discuss current approaches to treatment and management of MPS VI and MPS IVA, the infographics "Disease-modifying and supportive treatments for MPS VI/IVA" and "Surgical treatments for MPS IVA/VI symptoms" were developed (Figs. 5-8). The former discusses key information surrounding enzyme replacement therapy (ERT) and hematopoietic stem cell therapy (HSCT) in the context of MPS VI and MPS IVA, while introducing therapies aimed at addressing the symptoms of MPS. The latter infographic contains more information about select surgical interventions aimed at managing various symptoms of MPS VI and MPS IVA. 
One area of key patient and physician concern regarding MPS VI and MPS IVA was identified as the use of general anesthetics (GA) for surgical and investigative procedures. The infographics on "General anesthetics (GA) in MPS VI/IVA" describe the patient journey from before, during, and after surgeries or procedures involving GA (Figs. 9, 10). Respiratory and Ear-Nose-Throat (ENT) care in MPS VI and MPS IVA is addressed in the infographics "ENT and respiratory care for MPS VI/IVA" (Figs. 11, 12). This domain describes the impact of breathing difficulties on quality of life, and the supportive care that patients can receive from their treating HCPs.

\section{Discussion}

As patients are the key stakeholders for any disease, there is a clear need to communicate medical information and research in a manner that non-specialists will understand. This need is particularly apparent for complex diseases such as MPS IVA and MPS VI, for which detailed guidelines were recently released. While aimed at a specialist audience, these resources may be utilized by patients but also healthcare professionals without prior specialist knowledge of MPS diseases. Here, we have created visual infographics to relay the information from these guidelines that are most relevant to patients, and to provide an outline for how patient-friendly materials can be created for other diseases.

Clinical practice guidelines play an important role in educating HCPs and have been advocated as an essential part of good medical practice for several decades. When based on a systematic and critical review of the evidence, expert-led and consensus-driven guidelines can provide a powerful way to help translate the current body of knowledge into actual clinical practice, particularly in the setting of complex diseases $[17,18]$. However, specialist language and the technical complexity of such guidelines may represent a barrier to non-specialists that include patients and their caregivers as well as general HCPs.

In recognition of the need to communicate this information to patient lay audiences, there has been a rapid adoption of PLS among scientific publications in recent years. The results of a survey in 2018 highlighted the value of PLS for both patients and physicians. In this survey, a major theme that emerged in patient interviews was the importance of knowledge and the sense of empowerment it brings, with patients viewing PLS as tools to enable knowledge sharing and making important information accessible. Furthermore, physicians noted the value of PLS in opening patient dialog, saving time, and streamlining communication, as their patients were not completely dependent on their doctor for information [16].

For patients and their caregivers, accurate, reliable, and up-to-date information is thus essential to equip them to make informed choices about their care; however, such information on rare diseases is often lacking. In a survey of patients and caregivers conducted in the UK covering more than 450 rare diseases, nearly $70 \%$ of respondents reported feeling that they were not provided with enough information on their condition after diagnosis, and 35\% stated that they did not understand all the information they were given [19]. Similarly, in an analysis of the information needs of patients $(n=55)$ living with rare diseases and their relatives $(n=13)$ in Germany, interviewees cited a strong need for information after diagnosis on 
potential drug treatments and research, and a lack of practical information for everyday life issues [13]. The lack of such information can lead to feelings of resignation and fear among patients and their caregivers [13].

To meet these needs for PLS on the current management guidelines on MPS VI and MPS IVA, it was decided to use a visually appealing approach in the form of an infographic. An infographic is a visual diagram often used to convey complex information in a way that can be quickly and easily understood [20]. The use of infographics as a tool for summarizing and disseminating medical literature online and in print is increasing in popularity. Indeed, infographic-style summaries have been associated with higher reader preference and lower perceived mental effort during summary review compared with text-only summaries [21, 22]. In addition to the chosen medium of infographics, we developed a visual design theme utilizing a "superhero" motif. This design was chosen to engage and empower an adolescent to young adult audience.

Access to information that allows a patient and their caregiver to understand the cause of their rare disease, its symptoms, and impact is an important requirement for being able to cope with the disease in everyday life $[13,23]$. Patients who have access to understandable information around the treatment of their condition may also be in a better position to educate any non-expert HCPs they encounter, and thereby raise awareness of independently developed guidelines. As well as increasing the involvement of patients and caregivers in clinical care, engaging patients, caregivers, and patient advocacy groups in research can also play a key role in addressing evidence gaps for the management of rare diseases [11]. Furthermore, as a lack of information contributes to a lower than desired participation in clinical trials and other research into rare diseases [24], using PLS to increase awareness of relevant research projects being planned or conducted has the potential to increase the number of patients willing to participate.

Potential limitations associated with the development of these PLS for the MPS IVA and MPS VI guidelines should be acknowledged. Loss of nuance and/or precise detail may result from simplifying extensive technical texts. We hoped to minimize this issue by relying on an iterative approach to obtain alignment across the specialist members of our team and take in feedback at each step. Producing English language PLS may also be considered insufficient for a global patient audience from multiple cultural backgrounds and who may be non-fluent in English. However, our team included specialists based in a range of different countries aiming to reflect the global patient experience. Our use of plain language and clear images may also serve to lessen the issue of understandability for individuals who read English as a second or third language. However, the production of translated versions would represent an ultimate goal to obtain maximum audience reach, an approach that may be pursued in future with the materials presented here.

\section{Conclusions}

In conclusion, PLS have a central role to play in ensuring that the latest scientific research reaches wider audiences and we recommend that they become standard practice for pivotal publications such as 
disease management guidelines. The use of PLS to clearly convey the complex information included in such guidelines will ensure that patients and their caregivers are well informed about their disease and able to participate in decisions around their care in a more meaningful and constructive way.

\section{Abbreviations}

ARSB: $N$-acetyl-galactosamine-4-sulfatase (arylsulfatase B); CS: Chondroitin sulfate; CT: Computed tomography; ECG: Electrocardiogram; ENT: Ear-Nose-Throat; ERT: Enzyme replacement therapy; GA: General anesthetics; GAG: Glycosaminoglycan; GALNS: N-acetyl-galactosamine-6-sulfatase; HCPs: Healthcare professionals; HSCT: Hematopoietic stem cell therapy; IV: Intravenous; KS: Keratan sulfate; MDT: Multidisciplinary team; MPS: Mucopolysaccharidosis; MRI: Magnetic resonance imaging; PLS: Plain language summaries; TIVAD: Totally Implantable Venous Access Device.

\section{Declarations}

\section{Acknowledgements}

The authors would like to thank Terri Klein, President and Chief Executive Officer of the National MPS Society, Durham, North Carolina, United States of America, for her input during the initial stages of manuscript development, and the PAG UK MPS Society for their consultation on the design of the infographics. Medical writing and editorial support for the authors during the preparation of this manuscript was provided by William Jones, MBiochem, of Adelphi Communications Ltd., Adelphi Mill, Macclesfield, East Cheshire, United Kingdom. Financial support for these services was provided by BioMarin Pharmaceutical Inc.

\section{Authors' contributions}

All authors were involved in direction of the adaptation and design of the infographics presented in this manuscript. All authors reviewed and approved the final manuscript.

\section{Funding}

This manuscript was funded by BioMarin. BioMarin was not involved in any stage of content development for this manuscript and did not influence the design or content in any way. BioMarin was not included in any correspondence between the authors regarding this manuscript.

\section{Availability of data and materials}

Not applicable

\section{Ethics approval and consent to participate}

Not applicable 


\section{Consent for publications}

Not applicable

\section{Competing interests}

FSE has received consultancy and speaker fees from BioMarin, Takeda, and Sanofi Genzyme paid to their institution, travel grants from Pfizer and Sanofi Genzyme, and has participated as an investigator for clinical trials sponsored by Sobi and Sanofi Genzyme. WM has received speaker fees from BioMarin and Web MD Medscape paid to their institutional honorarium, received a Biobank research grant from BioMarin paid to their institution, and has acted as a non-paid consultant to Little People of America and the MPS Society on medical advisory boards. BS has received grants paid to the MPS Society from Takeda, BioMarin, and Regenxbio for speaker fees and payments. $\mathrm{CH}$ was employed by and owned shares in FYMCA Medical Ltd. until October 2020.

\section{References}

1. Muenzer J. Overview of the mucopolysaccharidoses. Rheumatology (Oxford). 2011;50 Suppl 5:412.

2. Coutinho MF, Lacerda L, Alves S. Glycosaminoglycan storage disorders: a review. Biochem Res Int. 2012;2012:471325.

3. Archer LD, Langford-Smith KJ, Bigger BW, Fildes JE. Mucopolysaccharide diseases: a complex interplay between neuroinflammation, microglial activation and adaptive immunity. J Inherit Metab Dis. 2014;37(1):1-12.

4. Leone A, Rigante D, Amato DZ, Casale R, Pedone L, Magarelli N, Colosimo C. Spinal involvement in mucopolysaccharidoses: a review. Childs Nerv Syst. 2015;31(2):203-12.

5. $\quad$ Cimaz R, La Torre F. Mucopolysaccharidoses. Curr Rheumatol Rep. 2014;16(1):389.

6. Valayannopoulos V, Nicely H, Harmatz P, Turbeville S. Mucopolysaccharidosis VI. Orphanet J Rare Dis. 2010;5(1):5.

7. Akyol MU, Alden TD, Amartino H, Ashworth J, Belani K, Berger Kl, et al. Recommendations for the management of MPS Vl: systematic evidence- and consensus-based guidance. Orphanet J Rare Dis. 2019;14(1):118.

8. Tomatsu S, Montaño AM, Oikawa H, Smith M, Barrera L, Chinen Y, et al. Mucopolysaccharidosis type IVA (Morquio A disease): clinical review and current treatment. Curr Pharm Biotechnol. 2011;12(6):931-45. 
9. Akyol MU, Alden TD, Amartino H, Ashworth J, Belani K, Berger Kl, et al. Recommendations for the management of MPS IVA: systematic evidence- and consensus-based guidance. Orphanet J Rare Dis. 2019;14(1):137.

10. Harmatz P, Mengel KE, Giugliani R, Valayannopoulos V, Lin SP, Parini R, et al. The Morquio A Clinical Assessment Program: baseline results illustrating progressive, multisystemic clinical impairments in Morquio A subjects. Mol Genet Metab. 2013;109(1):54-61.

11. Forsythe LP, Szydlowski V, Murad MH, Ip S, Wang Z, Elraiyah TA, et al. A systematic review of approaches for engaging patients for research on rare diseases. J Gen Intern Med. 2014;29(Suppl 3):S788-800.

12. Young K, Kaminstein D, Olivos A, Burroughs C, Castillo-Lee C, Kullman J, et al. Patient involvement in medical research: what patients and physicians learn from each other. Orphanet $\mathrm{J}$ Rare Dis. 2019;14(1):21.

13. Litzkendorf S, Babac A, Rosenfeldt D, Schauer F, Hartz T, Lührs V, et al. Information needs of people with rare diseases - what information do patients and their relatives require? Rare Disord Diagn Ther. 2016;2:2.

14. Uhlenbusch N, Löwe B, Depping MK. Perceived burden in dealing with different rare diseases: a qualitative focus group study. BMJ Open. 2019;9(12):e033353.

15. Nunn E, Pinfield S. Lay summaries of open access journal articles: engaging with the general public on medical research. Learn Publ. 2014;27:173-84.

16. Pushparajah DS, Manning E, Michels E, Arnaudeau-Bégard C. Value of developing plain language summaries of scientific and clinical articles: a survey of patients and physicians. Ther Innov Regul Sci. 2018;52(4):474-81.

17. Murad MH. Clinical Practice Guidelines: A primer on development and dissemination. Mayo Clin Proc. 2017;92(3):423-33.

18. Pavan S, Rommel K, Mateo Marquina ME, Höhn S, Lanneau V, Rath A. Clinical practice guidelines for rare diseases: The Orphanet Database. PLoS One. 2017;12(1):e0170365.

19. Muir E. The Rare Reality - an insight into the patient and family experience of rare disease. Rare Disease UK. 2016. Available at: https://www.raredisease.org.uk/media/1588/the-rare-reality-an-insightinto-the-patient-and-family-experience-of-rare-disease.pdf. Last accessed June 2021.

20. Balkac M, Ergun E. Role of infographics in healthcare. Chin Med J (Engl). 2018;131(20):2514-7.

21. Martin LJ, Turnquist A, Groot B, Huang SYM, Kok E, Thoma B, et al. Exploring the role of infographics for summarizing medical literature. Health Prof Edu. 2019;5(1):48-57. 
22. Martínez Silvagnoli L, Shepherd C, Pritchett J, Gardner J. How can we optimize the readability and format of plain language summaries for medical journal articles? A cross-sectional survey study. J Med Internet Res. 2020;22122. Available at: https://jmir.org/preprint/22122.

23. von der Lippe C, Diesen PS, Feragen KB. Living with a rare disorder: a systematic review of the qualitative literature. Mol Genet Genomic Med. 2017;5(6):758-73.

24. Boycott KM, Lau LP, Cutillo CM, Austin CP. International collaborative actions and transparency to understand, diagnose, and develop therapies for rare diseases. EMBO Mol Med. 2019;11(5):e10486.

25. European Medicines Agency. Naglazyme (galsulfase). Summary of product characteristics. 2020. Available at: https://www.ema.europa.eu/en/documents/product-information/naglazyme-eparproduct-information_en.pdf. Last accessed June 2021.

26. US Food and Drug Administration. Naglazyme (galsulfase). US prescribing information. 2019. Available at: https://www.accessdata.fda.gov/drugsatfda_docs/label/2019/125117s129lbl.pdf. Last accessed June 2021.

27. European Medicines Agency. Vimizim (elosulfase alfa). Summary of product characteristics. 2018. Available at: https://www.ema.europa.eu/en/documents/product-information/vimizim-eparproduct-information_en.pdf. Last accessed June 2021.

28. US Food and Drug Administration. Vimizim (elosulfase alfa). US prescribing information. 2019. Available at: https://www.accessdata.fda.gov/drugsatfda_docs/label/2019/125460s017lbl.pdf. Last accessed June 2021.

\section{Figures}


INTRODUCING YOUR CARE PROVIDERS: THE MULTIDISCIPLINARY TEAM (MDT)

A skilled multidisciplinary team (MDT) should be assembled to support you and help manage all the different ways that MPS VI can affect you

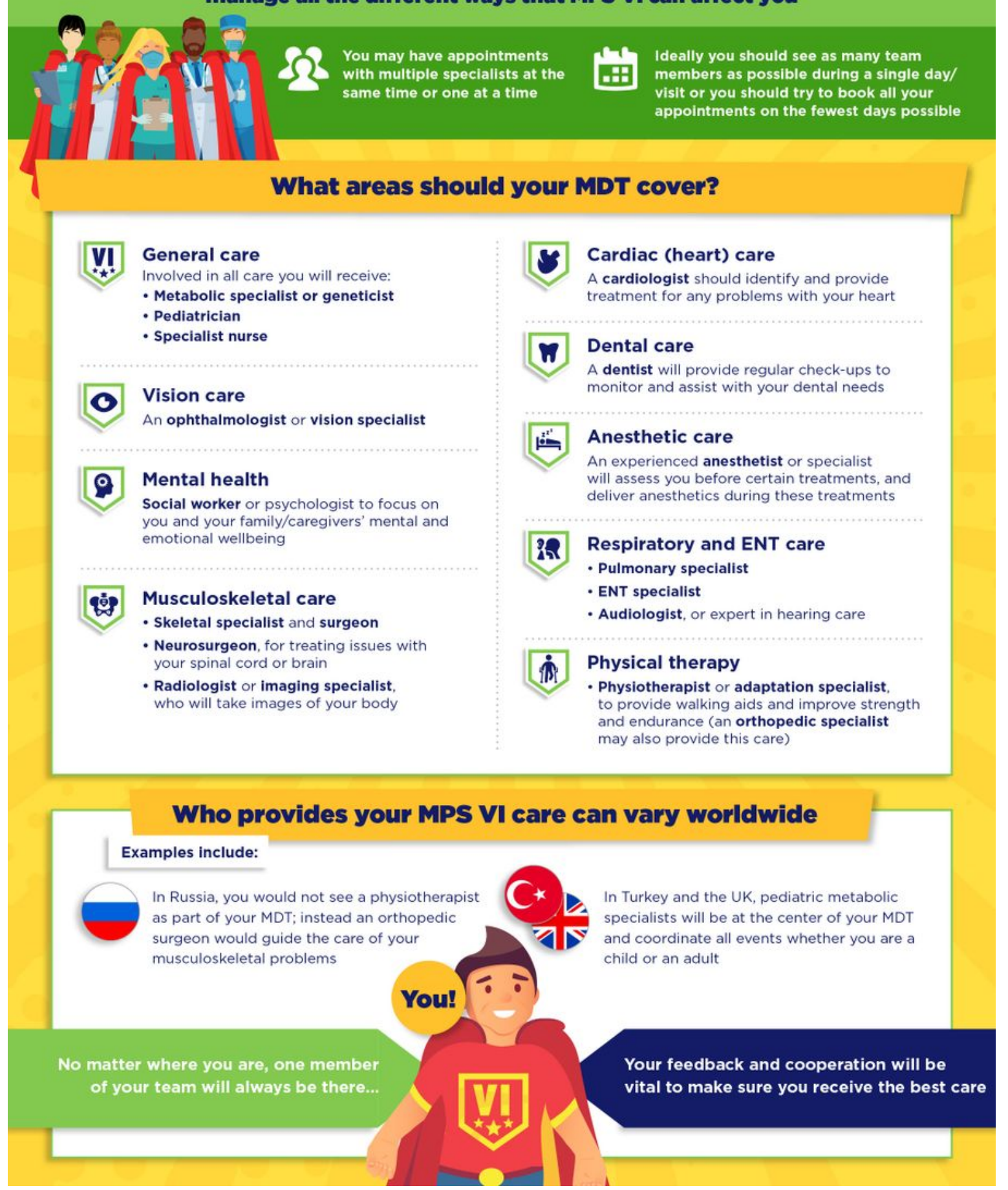

\section{Figure 1}

Introducing your care providers: The multidisciplinary team (MDT) in MPS VI infographic. This infographic provides a brief summary of key information found in the MPS VI treatment guidelines. For further details, please see the original paper published in the Orphanet Journal of Rare Diseases [7]. The overall Appraisal of Guidelines for Research and Evaluation (AGREE II) assessment score for the original guideline was 5.3/7 (where 1=lowest quality and 7=highest quality of guidance). 
INTRODUCING YOUR CARE PROVIDERS: THE MULTIDISCIPLINARY TEAM (MDT)

A skilled multidisciplinary team (MDT) should be assembled to support you and help manage all the different ways that MPS IVA can affect you

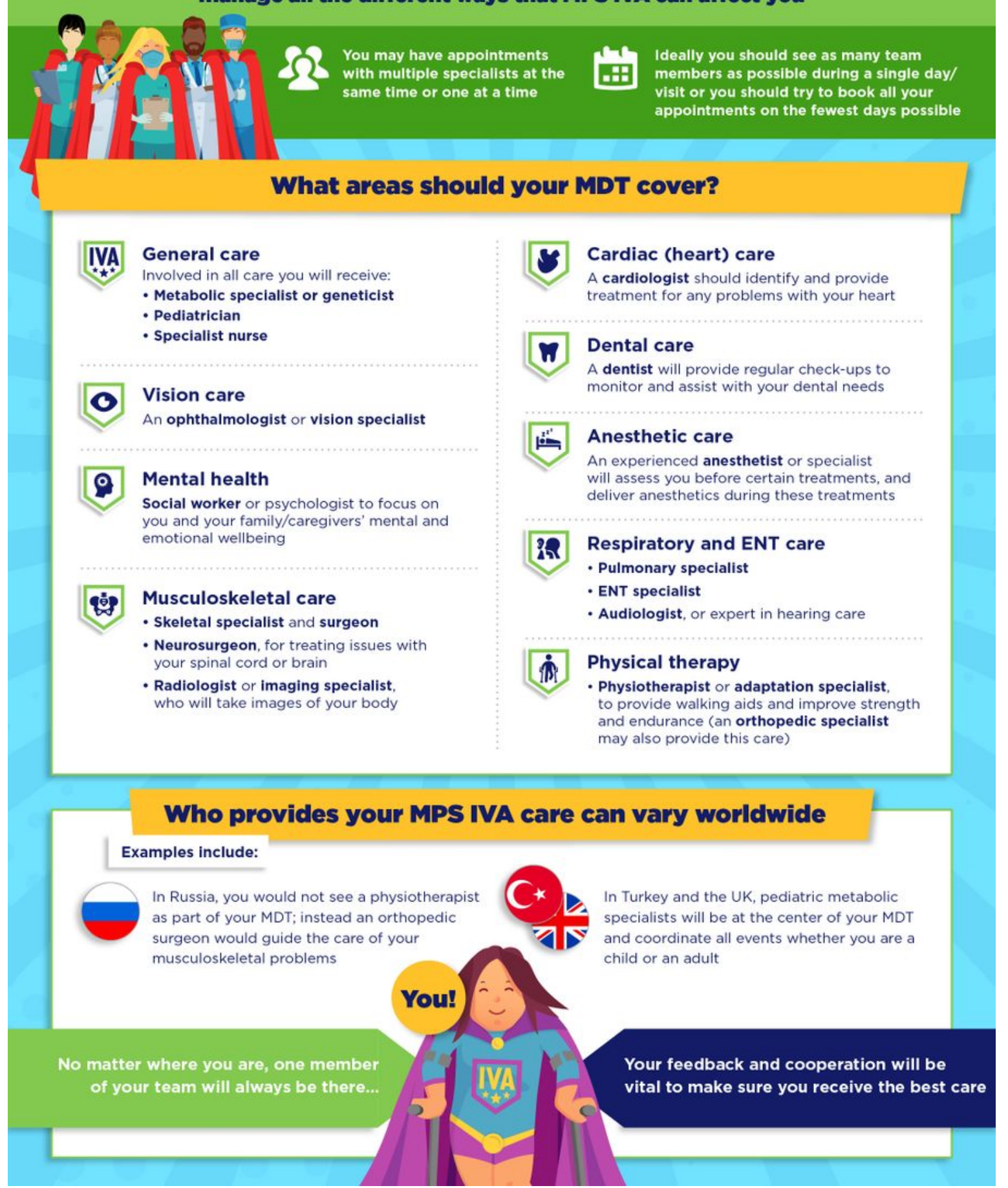

\section{Figure 2}

Introducing your care providers: The multidisciplinary team (MDT) in MPS IVA infographic. This infographic provides a brief summary of key information found in the MPS IVA treatment guidelines. For further details, please see the original paper published in the Orphanet Journal of Rare Diseases [9]. The overall Appraisal of Guidelines for Research and Evaluation (AGREE II) assessment score for the original guideline was 5.3/7 (where 1=lowest quality and 7=highest quality of guidance). 
(1) be diagnosed as early as possible A long-term plan
should be made
1) 通及

Regular tests given by a team of expert doctors is a vital part of any MPS VI plan

What are the tests and how often should they be done?

A full range of the below tests should be carried out at diagnosis, then regular tests

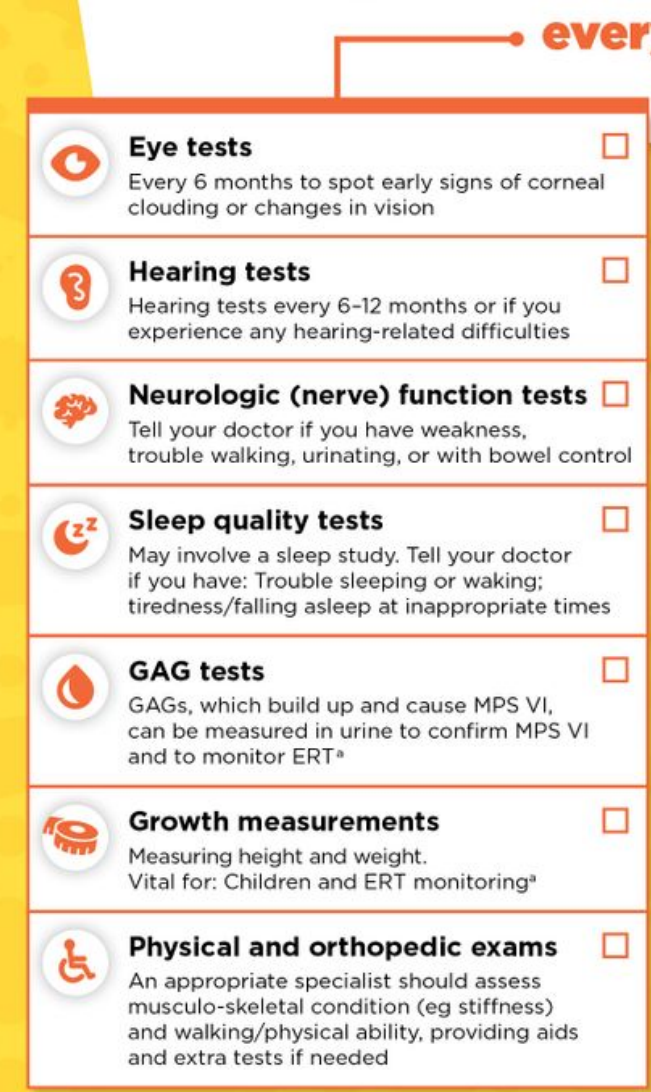

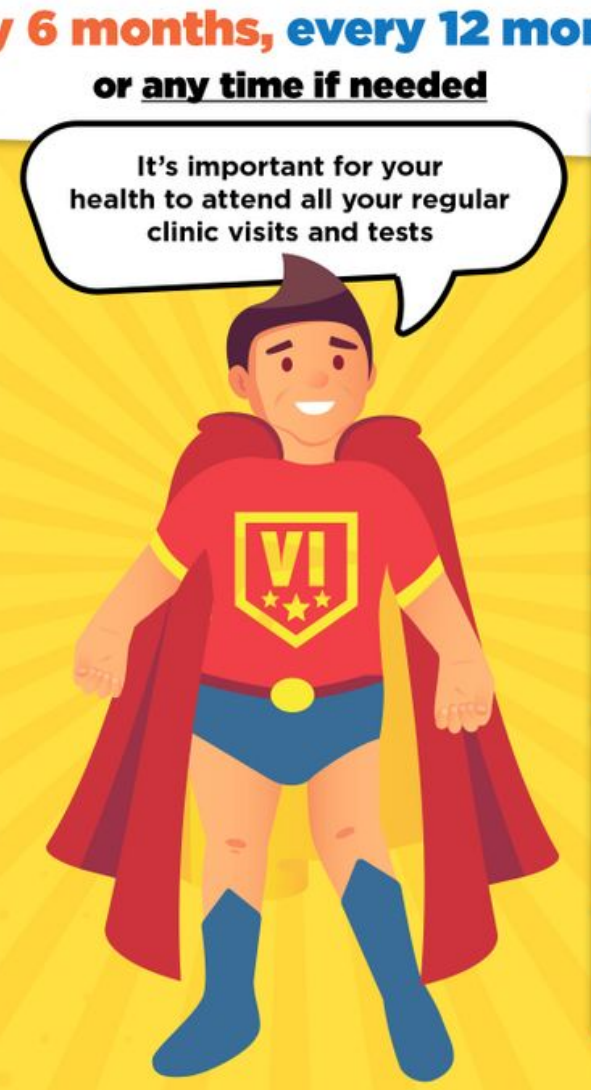

nths,

\section{Dental health check-ups \\ To ensure healthy teeth and provide special toothpaste and/or dental hygiene tools \\ 9. ENT exams \\ An exam using a small camera to "map" your airway. At any time if you have issues breathing or shortness of breath and feel you need help, tell your doctor}

\section{(1) Heart function tests ${ }^{b}$}

Tests to check your heart is working correctly: Echocardiogram, 12-lead ECG, Holter/event monitoring ECG

\section{* Radiology exams}

Intermittent X-rays of the spine in your neck, back, and your legs, and annual MRI scans to give doctors an inside view of your body

\section{Endurance tests}

Checks for issues with walking using one of two tests:

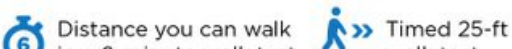
in a 6-minute walk test ..... walk test

\section{Disease burden questionnaires}

Questions about how your condition affects your general life (eg everyday activities)

\section{Figure 3}

Regular tests and check-ups for MPS VI infographic. This infographic provides a summary of key information found in the MPS VI treatment guidelines. For further details, please see the original paper published in the Orphanet Journal of Rare Diseases [7]. The overall Appraisal of Guidelines for Research and Evaluation (AGREE II) assessment score for the original guideline was 5.3/7 (where $1=$ lowest quality and $7=$ highest quality of guidance). aPlease see the companion infographic "Disease-modifying and supportive treatments for MPS VI" for more information about ERT. bTime between heart function tests could be longer if no issues are found. 
(1) be diagnosed as early as possible

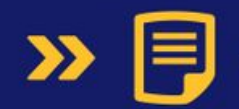

A long-term plan should be made
1) 通及

Regular tests given by a team of expert doctors is a vital part of any MPS IVA plan

What are the tests and how often should they be done?

A full range of the below tests should be carried out at diagnosis, then regular tests - every 6 months, every 12 months,

\begin{tabular}{|c|c|}
\hline & $\begin{array}{l}\text { Eye tests } \\
\text { Every } 6 \text { months to spot any cloudy vision }\end{array}$ \\
\hline & $\begin{array}{l}\text { Hearing tests } \\
\text { Hearing tests every } 6-12 \text { months or if you } \\
\text { experience any hearing-related difficulties }\end{array}$ \\
\hline & $\begin{array}{l}\text { Neurologic (nerve) function tests } \\
\text { Tell your doctor if you have weakness, } \\
\text { trouble walking, urinating, or with bowel contro }\end{array}$ \\
\hline & $\begin{array}{l}\text { Sleep quality tests } \\
\text { May involve a sleep study. Tell your doctor } \\
\text { if you have: Trouble sleeping or waking: } \\
\text { tiredness/falling asleep at inappropriate times }\end{array}$ \\
\hline & $\begin{array}{l}\text { GAG tests } \\
\text { GAGs, which build up and cause MPS IVA, } \\
\text { can be measured in urine to confirm MPS IVA } \\
\text { and to monitor ERT }\end{array}$ \\
\hline & $\begin{array}{l}\text { Growth measurements } \\
\text { Measuring height, weight, and puberty. } \\
\text { Vital for: Children and ERT monitoring }\end{array}$ \\
\hline & $\begin{array}{l}\text { Physical and orthopedic exams } \\
\text { An appropriate specialist should assess } \\
\text { musculo-skeletal condition (eg laxity) } \\
\text { and walking/physical ability, providing aids } \\
\text { and extra tests if needed }\end{array}$ \\
\hline
\end{tabular}

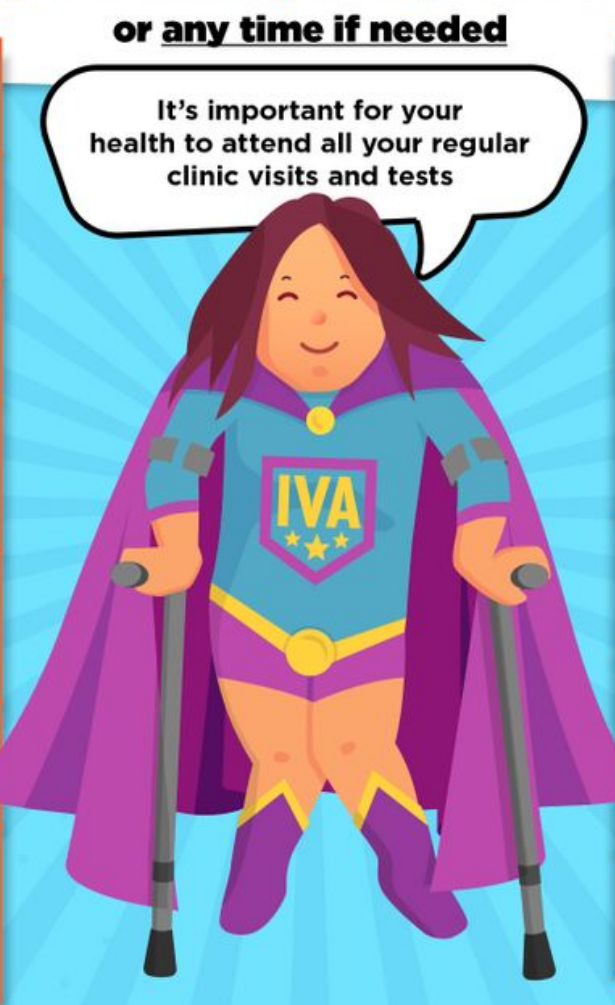

\begin{tabular}{|l|}
\hline Dental health check-ups \\
To ensure healthy teeth and provide special \\
toothpaste and/or dental hygiene tools
\end{tabular}

\section{Figure 4}

Regular tests and check-ups for MPS IVA infographic. This infographic provides a summary of key information found in the MPS IVA treatment guidelines. For further details, please see the original paper published in the Orphanet Journal of Rare Diseases [9]. The overall Appraisal of Guidelines for Research and Evaluation (AGREE II) assessment score for the original guideline was 5.3/7 (where $1=$ lowest quality and $7=$ highest quality of guidance). aPlease see the companion infographic "Disease-modifying and supportive treatments for MPS IVA" for more information about ERT. bTime between heart function tests could be longer if no issues are found. 
DISEASE-MODIFYING AND SUPPORTIVE TREATMENTS FOR MPS VI

Disease-Modifying Treatments

Aim to "modify" the course of MPS VI and slow down its worsening

\section{ERT (Galsulfase)}

ERT involves treatment with the drug galsulfase,

which substitutes for the faulty enzyme arylsulfatase B and prevents the build-up of GAGs

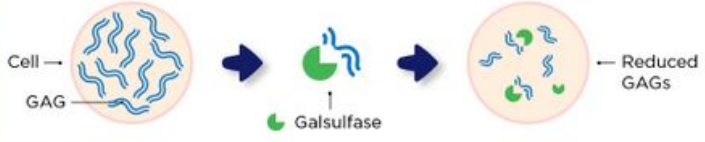

If you have been newly diagnosed and could benefit, you should start taking ERT as soon as possible

Studies have shown ERT can help Improve:
4. Exercise
ability
Breathing
Dally activities

You may have some side effects with ERT, including:

of

Headache, fever

(4)

First ERT treatment in hospital to make sure it goes well Discuss if later treatments can be done at home

ERT is given as a slow injection (infusion) every week

\section{HSCT}

An injection of special cells that can make the missing arylsulfatase B enzyme, and so aims to restore your body's ability to break down GAGs

May be recommended in special circumstances and if a suitable stem cell donor is available

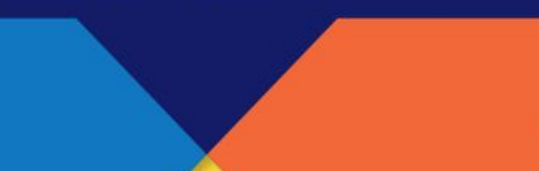

Supportive Treatments

Aim to help correct and ease the symptoms of MPS VI

\section{Treatments}

Hip surgery

Reconstructive surgery/hip replacement

Spinal surgery

\section{Knock knee surgery}

Heart surgery

\section{Craniovertebral surgery}

Surgery on the spine leading up to your skull to treat spinal cord injury

\section{Surgeries on your airway}

Hearing treatment, eg hearing aids

\section{Dental treatments}

Surgery to put in a "line" for ERT

See more about supportive surgeries In the companion Infographic "Surgical Treatments for MPS VI Symptoms"

\section{Figure 5}

Disease-modifying and supportive treatments for MPS VI infographic. This infographic provides a summary of key information found in the MPS VI treatment guidelines. For further details, please see the original paper published in the Orphanet Journal of Rare Diseases [7]. The overall Appraisal of Guidelines for Research and Evaluation (AGREE II) assessment score for the original guideline was 5.3/7 (where $1=$ lowest quality and $7=$ highest quality of guidance). aThe prescribing information for Naglazyme (galsulfase) contains warnings for anaphylaxis and severe allergic reactions, which have been reported in clinical studies $[25,26]$. Medical support should be present during all treatments. If these reactions occur, ERT infusion should be immediately halted and treatment given. 
Disease-Modifying Treatments

Aim to "modify" the course of MPS IVA and slow down its worsening

\section{ERT (Elosulfase Alfa)}

ERT involves treatment with the drug elosulfase alfa, which substitutes for the faulty enzyme GALNS and prevents the build-up of GAGs

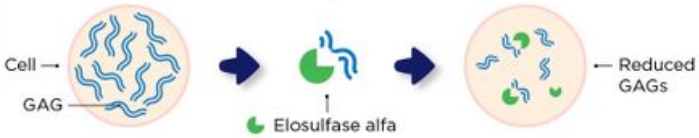

If you have been newly diagnosed and could benefit, you should start taking ERT as soon as possible

Studies have shown ERT can help Improve:
4. Exercise
ability
Breathing
Dally activities

You may have some side effects with ERT, Including:

of

Headache, fever

$\mathrm{S}_{4}$

RT, Including:

a

people'

First ERT treatment in hospital to make sure it goes well Discuss if later treatments can be done at home

ERT is given as a slow injection (infusion) every week

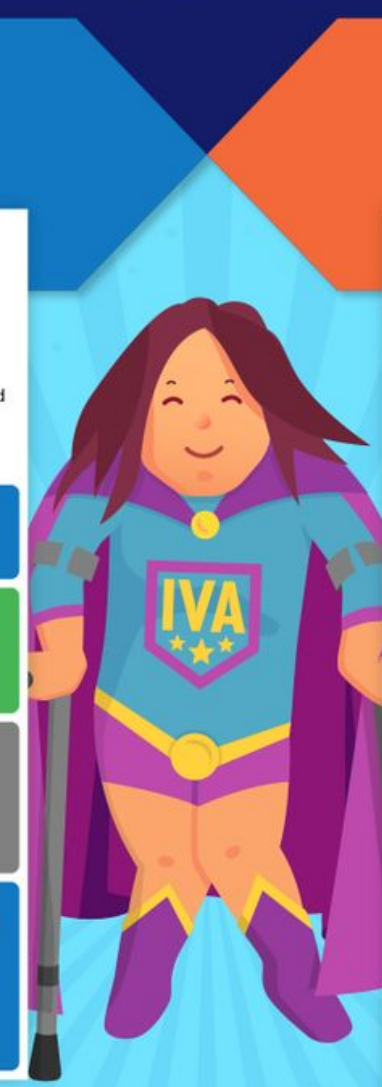

Stem cell therapy (HSCT) is not recommended

An injection of special cells that can make the missing GALNS enzyme, and

so aims to restore your body's ability to break down GAGs. However. HSCT is not well studied in MPS IVA and carries risk of serious harm including death
Supportive Treathents

Aim to help correct and ease the symptoms of MPS IVA

\section{Treatments}

Hip surgery

Reconstructive surgery/hip replacement

\section{Spinal surgery}

\section{Knock knee surgery}

\section{Heart surgery}

\section{Craniovertebral surgery}

Surgery on the spine leading up to your skull to treat spinal cord injury

\section{Surgeries on your airway}

Hearing treatment, eg hearing aids

\section{Dental treatments}

\section{Surgery to put in a "line" for ERT}

See more about supportive surgeries in the companion infographic "Surgical Treatments for MPS IVA Symptoms"

\section{Figure 6}

Disease-modifying and supportive treatments for MPS IVA infographic. This infographic provides a summary of key information found in the MPS IVA treatment guidelines. For further details, please see the original paper published in the Orphanet Journal of Rare Diseases [9]. The overall Appraisal of Guidelines for Research and Evaluation (AGREE II) assessment score for the original guideline was 5.3/7 (where $1=$ lowest quality and $7=$ highest quality of guidance). aThe prescribing information for Vimizim (elosulfase alfa) contains warnings for anaphylaxis and severe allergic reactions, which have been reported in clinical studies $[27,28]$. Medical support should be present during all treatments. If these reactions occur, ERT infusion should be immediately halted and treatment given. 


\section{SURGICAL TREATMENTS FOR MPS VI SYMPTOMS}

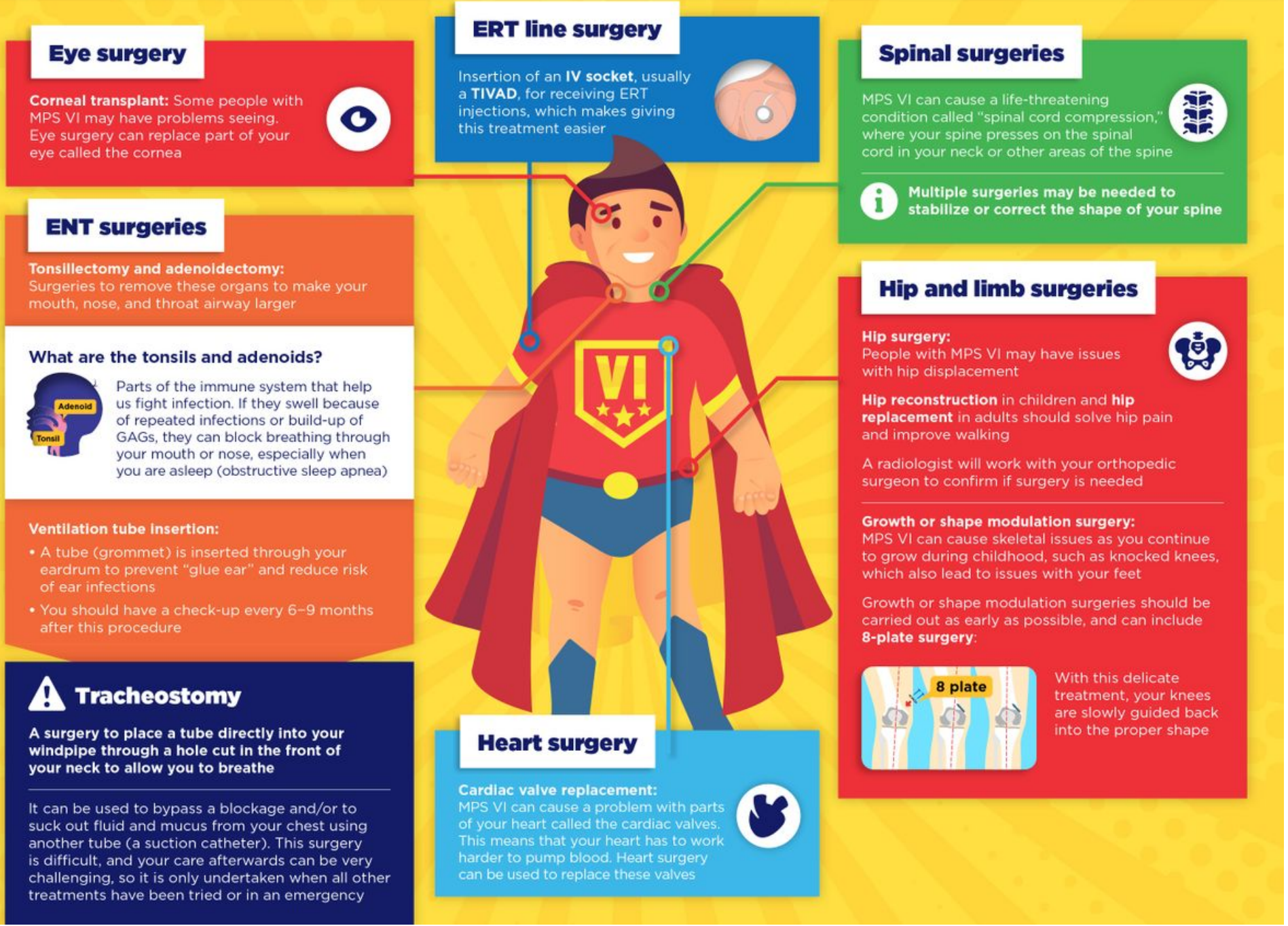

\section{Figure 7}

Surgical treatments for MPS VI symptoms infographic. This infographic provides a summary of key information found in the MPS VI treatment guidelines. For further details, please see the original paper published in the Orphanet Journal of Rare Diseases [7]. The overall Appraisal of Guidelines for Research and Evaluation (AGREE II) assessment score for the original guideline was 5.3/7 (where 1=lowest quality and $7=$ highest quality of guidance). 


\section{ENT surgeries}

Tonsillectomy and adenoldectomy:

Surgeries to remove these organs to make your

mouth, nose, and throat airway larger

What are the tonsils and adenoids?

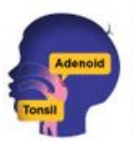

Parts of the immune system that help us fight infection. If they swell because of repeated infections or build-up of GAGs, they can block breathing through your mouth or nose, especially when you are asleep (obstructive sleep apnea)

\section{Ventilation tube insertion:}

- A tube (grommet) is inserted through your eardrum to prevent "glue ear" and reduce risk of ear infections

You should have a check-up every 6-9 months after this procedure

\section{! Tracheostomy}

A surgery to place a tube directly into your windpipe through a hole cut in the front of your neck to allow you to breathe

It can be used to bypass a blockage and/or to suck out fluid and mucus from your chest using another tube (a suction catheter). This surgery is difficult, and your care afterwards can be very challenging, so it is only undertaken when all other treatments have been tried or in an emergency

\section{ERT line surgery}

Insertion of an IV socket, usually a TIVAD, for receiving ERT injections, which makes giving this treatment easier

\section{Spinal surgeries}

MPS IVA can cause a life-threatening condition called "spinal cord compression cord in your neck or other areas of the spine

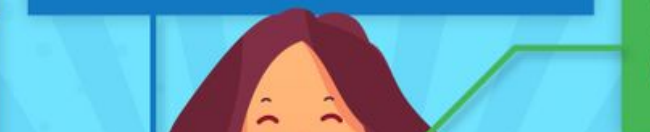

Multiple surgeries may be needed to stabilize or correct the shape of your spine

\section{Hip and limb surgeries}

Hip surgery:

People with MPS IVA may have issues with hip displacement

Hip reconstruction in children and hip replacement in adults should solve hip pain and improve walking

A radiologist will work with your orthopedic surgeon to confirm if surgery is needed

Growth or shape modulation surgery: MPS IVA can cause skeletal issues as you continue to grow during childhood, such as knocked knees, which also lead to issues with your feet

Growth or shape modulation surgeries should be carried out as early as possible, and can include 8-plate surgery.

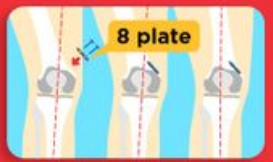

With this delicate treatment, your knees are slowly guided back into the proper shape

\section{Heart surgery}

Cardiac valve replacement: Heart surgery to replace parts of your heart called the cardiac valves

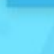

\section{Figure 8}

Surgical treatments for MPS IVA symptoms infographic. This infographic provides a summary of key information found in the MPS IVA treatment guidelines. For further details, please see the original paper published in the Orphanet Journal of Rare Diseases [9]. The overall Appraisal of Guidelines for Research and Evaluation (AGREE II) assessment score for the original guideline was 5.3/7 (where $1=$ lowest quality and $7=$ highest quality of guidance). 


\section{GENERAL ANESTHETICS (GAS) IN MPS VI}

People with MPS VI, like you, will likely need various surgeries and hospital procedures as part of their treatment. These can include:
(3)
Surgeries on
your skeleton

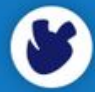
Heart
surgery
(6) Surgery on
(6)
Surgery on your eyes
CT and MRI
scans if you
cannot lie still

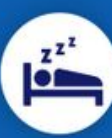

For these treatments you may be given a general anesthetic (GA), gases, and/or drugs that

keep you asleep and pain free, and a tube placed in your windpipe to help you breathe

\section{Remember: Specialist care needs a specialist center}

GAs can pose serious risks in people with MPS VI, but these can be minimized if the correct care is given at every stage by a team of expert doctors

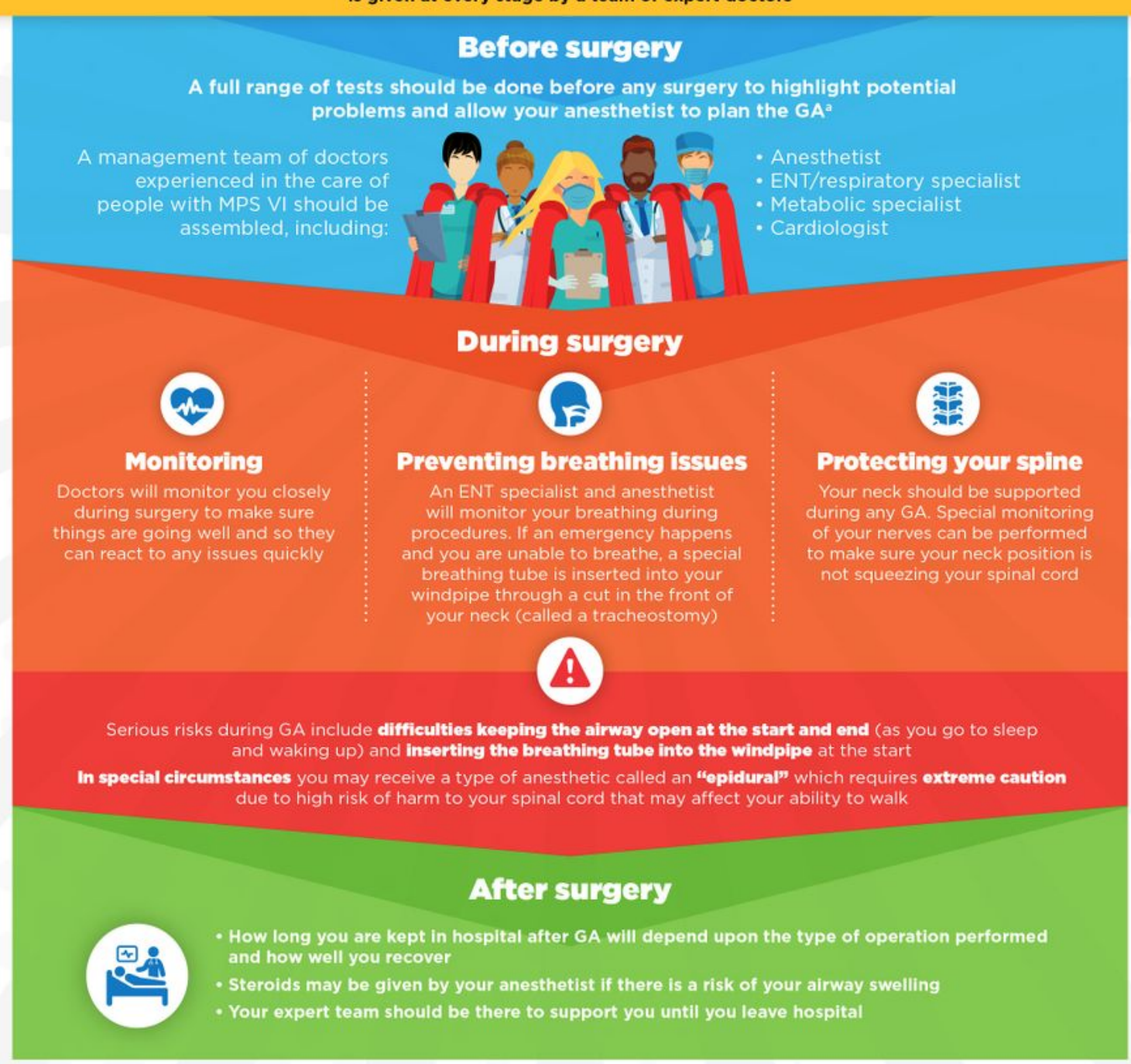

Figure 9

General anesthetics (GAs) in MPS VI infographic. This infographic provides a summary of key information found in the MPS VI treatment guidelines. For further details, please see the original paper published in the Orphanet Journal of Rare Diseases [7]. The overall Appraisal of Guidelines for Research and Evaluation (AGREE II) assessment score for the original guideline was 5.3/7 (where 1=lowest quality 
and 7=highest quality of guidance). aFor more information on the range of tests that should be carried out, please see the companion infographic "Regular tests and check-ups for MPS VI".

\section{GENERAL ANESTHETICS (GAS) IN MPS IVA}

People with MPS IVA, like you, will likely need various surgeries and hospital procedures as part of their treatment. These can include:
(3)
Surgeries on
your skeleton
(6)
Surgery on
your airway

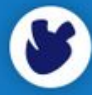
Heart
surgery
(6)
Surgery on
your eyes

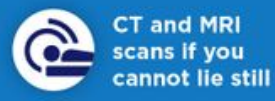

(⿻)

For these treatments you may be given a general anesthetic (GA), gases, and/or drugs that

keep you asleep and pain free, and a tube placed in your windpipe to help you breathe

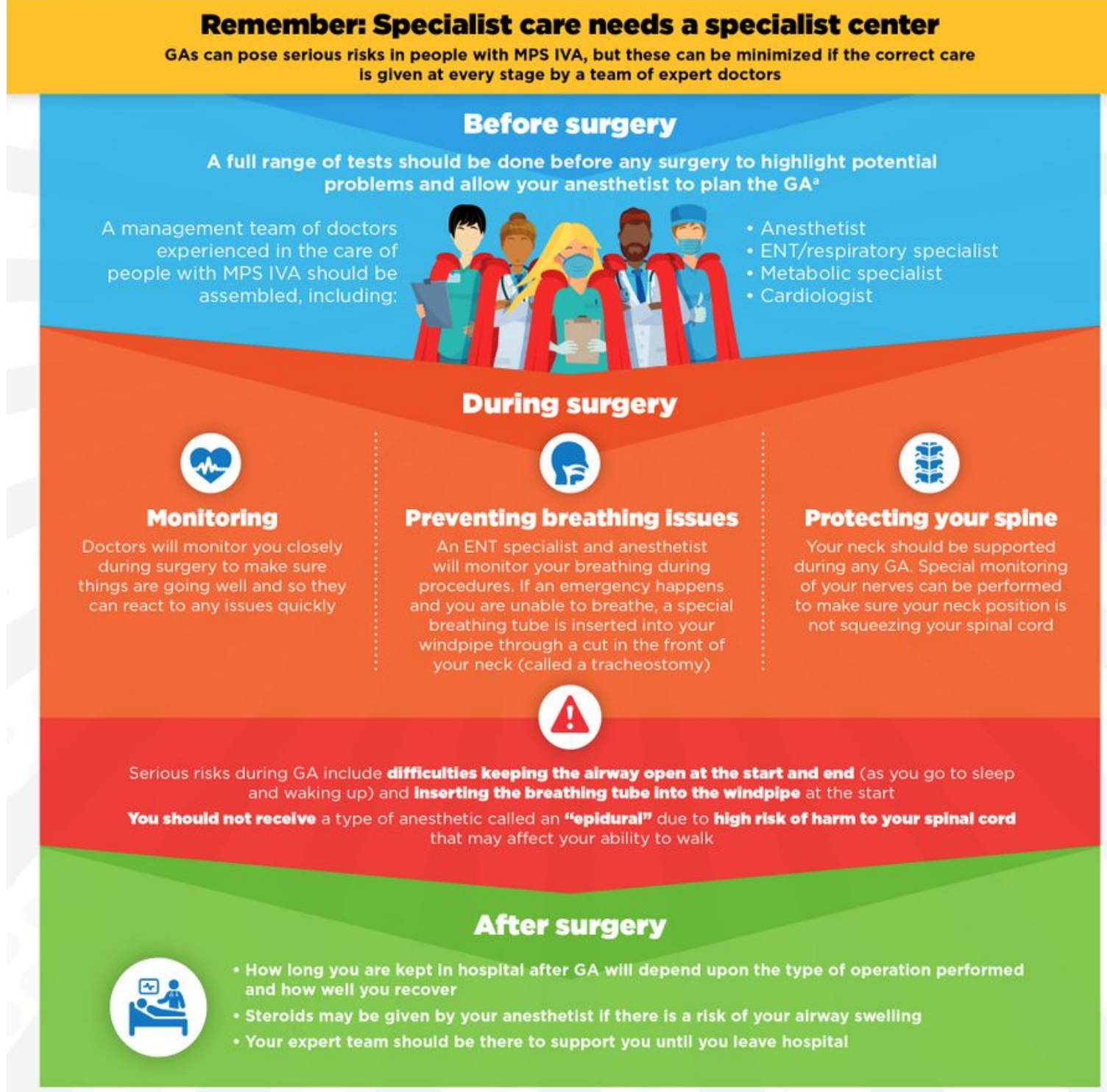

Figure 10

General anesthetics (GAs) in MPS IVA infographic. This infographic provides a summary of key information found in the MPS IVA treatment guidelines. For further details, please see the original paper published in the Orphanet Journal of Rare Diseases [9]. The overall Appraisal of Guidelines for Research 
and Evaluation (AGREE II) assessment score for the original guideline was 5.3/7 (where 1=lowest quality and $7=$ highest quality of guidance). aFor more information on the range of tests that should be carried out, please see the companion infographic "Regular tests and check-ups for MPS IVA".

\section{ENT AND RESPIRATORY CARE FOR MPS VI}

\section{MPS VI and your airway}

Your nose, windpipe, and lungs can be affected by MPS VI

Issues with growth can result in your windpipe growing longer than your chest space causing it to kink/bend

The health and stability of the spine in your neck is also important as damage to your spinal cord can affect your breathing

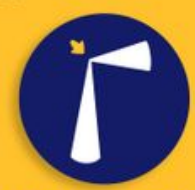

Kinks and bends cause blockages

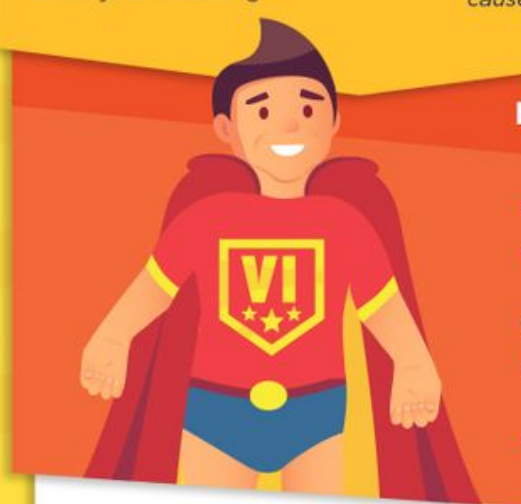

\section{How might this affect you?}

- Difficulty breathing during exercise

- Disturbed breathing, including snoring and breath-holding (apnea), during sleep

- Need for speclal measures during surgerles"

- Blockages in different parts of your airway that can get worse over time requiring neck extension

- Hearing loss and/or ear infections

MPS VI and your ears

wo main ways:

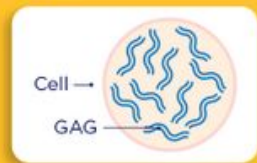

GAGs can build up and damage the cells in your ear that allow you to hear

\section{What can your care providers do to help?}

Treatments to help with your sleeping

\section{$x^{x^{2}} \quad$ Sleep study}

If you feel tired, aren't getting enough sleep, are falling asleep inappropriately, or even have bad breath, your doctor may recommend an overnight sleep study to help identify any issues

Constant Positive Airway Pressure (CPAP) therapy A device with a mask you wear during sleeping which provides air at increased pressure to hold open your airway and prevent blockages

Non-Invasive Positive Pressure Ventilation (NIPPV) Another option if CPAP is not working for you, which works in a similar way

Supplemental oxygen may be prescribed after CPAP or NIPPV if you are still not getting as much oxygen as you need during sleep

When receiving night-time oxygen, you should be monitored for side effects such as shortness of breath in the day

Vaccinations

You should receive a regular flu vaccine and vaccines for other diseases that can seriously worsen your breathing
Surgical treatments

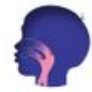

Tonsillectomy and adenoidectomy

Removal of your tonsils or adenoids to open airways and improve your breathing

Ventilation tube (grommet) insertion

Insertion of a tube (grommet) into your ear and through your eardrum to prevent glue ear and reduce your risk of ear infections

Grommets are temporary and may not be the best way to manage hearing loss due to glue ear over longer periods

Surgical treatments

A hearing aid amplifies the sound picked up by your ears and provides long-term management for loss of hearing due to glue ear

In special cases, surgery to insert a bone-anchored or cochlear hearing implant may be suggested

\section{Figure 11}

ENT and respiratory care for MPS VI infographic. This infographic provides a summary of key information found in the MPS VI treatment guidelines. For further details, please see the original paper 
published in the Orphanet Journal of Rare Diseases [7]. The overall Appraisal of Guidelines for Research and Evaluation (AGREE II) assessment score for the original guideline was 5.3/7 (where 1=lowest quality and 7=highest quality of guidance). aSee the companion infographic "General anesthetics for MPS VI" for more information.

\section{ENT AND RESPIRATORY CARE FOR MPS IVA}

\section{MPS IVA and your airway}

Your nose, windpipe, and lungs can be affected by MPS IVA

Issues with growth can result in your windpipe growing longer than your chest space causing it to kink/bend

The health and stability of the spine in your neck is also important as damage to your spinal cord can affect your breathing

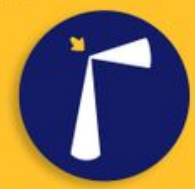

Kinks and bends cause blockages

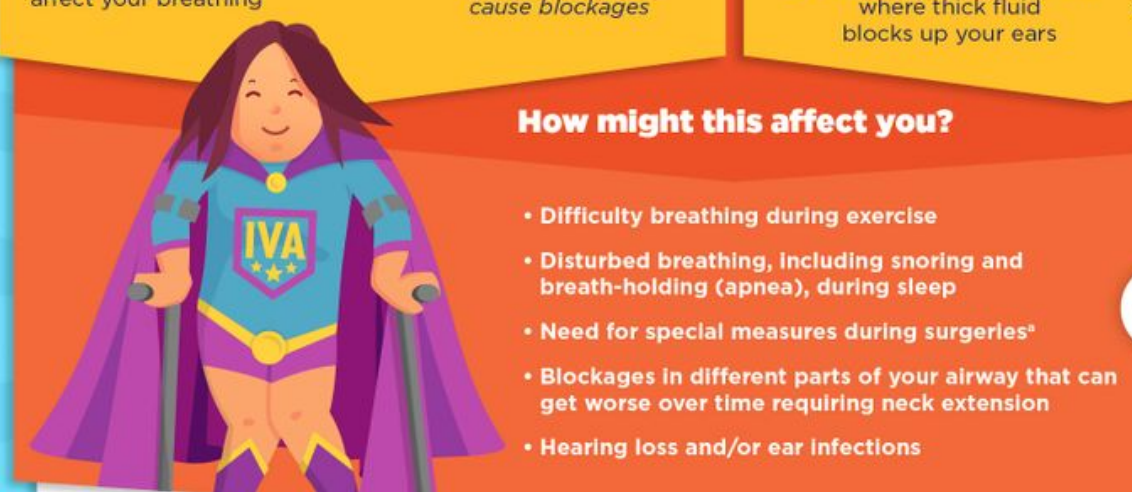

MPS IVA and your ears

Your ears can be affected by MPS IVA in two main ways:

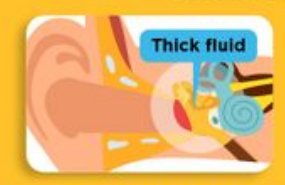

A condition called "glue ear," where thick fluid blocks up your ears

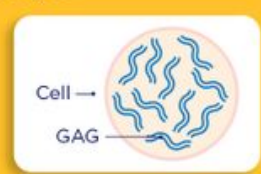

GAGs can build up and damage the cells in your ear that allow you to hear

What can your care providers do to help?

Treatments to help with your sleeping

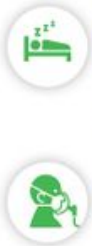

\section{Sleep study}

If you feel tired, aren't getting enough sleep, are falling asleep inappropriately, or even have bad breath, your doctor may recommend an overnight sleep study to help identify any issues

Constant Positive Airway Pressure (CPAP) therapy A device with a mask you wear during sleeping which provides air at increased pressure to hold open your airway and prevent blockages

Non-Invasive Positive Pressure Ventilation (NIPPV) Another option if CPAP is not working for you, which works in a similar way

Supplemental oxygen may be prescribed after CPAP or NIPPV if you are still not getting as much oxygen as you need during sleep

When receiving night-time oxygen, you should be monitored for side effects such as shortness of breath in the day

Vaccinations

You should receive a regular flu vaccine and vaccines for other diseases that can seriously worsen your breathing
Surgical treatments

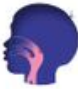

Tonsillectomy and adenoidectomy

Removal of your tonsils or adenoids to open

airways and improve your breathing

Ventilation tube (grommet) insertion

Insertion of a tube (grommet) into your ear and through your eardrum to prevent glue ear and reduce your risk of ear infections

Grommets are temporary and may not be the best way to manage hearing loss due to glue ear over longer periods

Surgical treatments

A hearing aid amplifies the sound picked up by your ears and provides long-term management for loss of hearing due to glue ear

In special cases, surgery to insert a bone-anchored or cochlear hearing implant may be suggested 
ENT and respiratory care for MPS IVA infographic. This infographic provides a summary of key information found in the MPS IVA treatment guidelines. For further details, please see the original paper published in the Orphanet Journal of Rare Diseases [9]. The overall Appraisal of Guidelines for Research and Evaluation (AGREE II) assessment score for the original guideline was 5.3/7 (where 1=lowest quality and 7=highest quality of guidance). aSee the companion infographic "General anesthetics for MPS IVA" for more information. 